\title{
LIMIT SET EQUIVALENCES OF REPLETE SEMIGROUPS
}

\section{ETHAN M. COVEN AND WILLIAM L. REDDY}

1. Introduction and definitions. Consider a one-parameter flow $(X, T)$. There are two classes of replete semigroups properly contained in $T$, positive (containing positive elements) and negative. If $P$ is positive, then the $P$-limit set $P_{x}$ of $x$ (defined below) is nothing but $\omega(x)$ and if $P$ is negative, the $P$-limit set of $x$ is $\alpha(x)$. In fact, if we compactify $T$ by the addition of $+\infty$ and $-\infty$, and let $T$ fix these two points and translate the others, the result is a transformation group such that $P$ is positive if and only if $P_{0}=\omega(0)$. In this paper we consider generalizations of this situation to the case of limit sets of replete semigroups in a locally compact abelian group. There are two natural generalizations of $\omega(x)$ available. See $[2, \S 2]$ for a discussion of this point. Related questions are studied in [1], [2], [5].

Throughout this paper, the terminology of [3] will be used. $(X, T, \pi)$ will denote a transformation group where $T$ is locally compact and abelian. The letter $e$ will denote the identity of $T$, and $P$ and $Q$ will denote replete semigroups properly contained in $T$. A subset $S$ of $T$ will be called $P$-extensive if it meets $p P$ for each $p \in P$, weakly $P$-extensive if it meets each replete semigroup contained in $P$, and $P$-syndetic if $P \subseteq S K$ for some compact set $K$. The $P$-limit set $P_{x}$ of a point $x \in X$ is defined by

$$
P_{x}=\bigcap_{p \in P} \mathrm{Cl}_{x} x p P .
$$

The weak $P$-limit set $P(x)$ of $x$ is defined by

$$
P(x)=\cap \mathrm{Cl}_{\boldsymbol{x}} x Q
$$

where the intersection is taken over all replete semigroups $Q \subseteq P$. If $T$ is the integers or the reals, then $P_{x}=P(x)$. We will say that $P$ and $Q$ have the same syndetic (extensive, weakly extensive) sets provided that each subset $S$ of $T$ has the property that $S \cap P$ is $P$-syndetic ( $P$ extensive, weakly $P$-extensive) if and only if $S \cap Q$ is $Q$-syndetic ( $Q$-extensive, weakly $Q$-extensive). If only replete semigroups are required to have this property, we will say that $P$ and $Q$ have the same syndetic (extensive, weakly extensive) replete semigroups.

In $\$ 2$ we investigate an equivalence relation among replete semigroups in $T$ whose classes turn out to consist of precisely those replete

Received by the editors April 18, 1969. 
semigroups which have identical limit sets for every transformation group, or (equivalently) have the same extensive sets, or (equivalently) have the same syndetic sets. In $\$ 3$ we study an equivalence relation whose classes consist of those replete semigroups having the same weak limit sets or (equivalently) the same extensive replete semigroups, or (equivalently) the same weakly extensive sets.

2. Strong equivalence. We say that $P$ and $Q$ are strongly equivalent and write $P \approx Q$ provided that there exist $p \in P$ and $q \in Q$ such that $p P \subseteq Q$ and $q Q \subseteq P$.

It is easy to see that this is an equivalence relation. It follows immediately from the definition that $p P \approx P$ for each $p \in P$ and that if $Q \subseteq P$, then $P \approx Q$ if and only if there is $p \in P$ such that $p P \subseteq Q$. It follows from this and from the transitivity of strong equivalence that $P \approx Q$ if and only if given $p_{0} \in P$ and $q_{0} \in Q$, there exist $p \in P$ and $q \in Q$ such that $p P \subseteq q_{0} Q$ and $q Q \subseteq p_{0} P$.

Lemma 1. The interior of $P\left(\right.$ denoted $\left.P^{0}\right), \bar{P}$ and $P \cup\{e\}$ are all replete semigroups strongly equivalent to $P$.

Proof. $P^{0}$ and $\bar{P}$ are semigroups by the continuity of the group operation, and $P \cup\{e\}$ is obviously a semigroup. By [2, Lemma 2.6], $P^{0}$ is replete, and both $\bar{P}$ and $P \cup\{e\}$ are replete since both contain $P$.

Let $p \in P^{0}$. If $p P \Phi P^{0}$, then there exists $p^{\prime} \in P$ and an open neighborhood $N$ of $e$ such that $p N \subseteq P^{0}$ and $p p^{\prime} N \cap P^{0}=\varnothing$. But then $p p^{\prime} N \subseteq p^{\prime} P^{0} \subseteq P^{0}$ and so $p p^{\prime} N \subseteq P^{0}$ which is a contradiction. Thus $P \approx P^{0}$.

Let $K$ be a compact neighborhood of $e$. Then $\bar{P} \subseteq P K$ and there exists $p \in P$ such that $p K \subseteq P$. Hence $p \bar{P} \subseteq p P K \subseteq P$ and so $P \approx \bar{P}$.

Since $p(P \cup\{e\}) \subseteq P$ for any $p \in P$, we have $P \approx P \cup\{e\}$.

REMARK. If $S$ is a replete subset of $T$ and if $K$ is a compact subset of $T$, then there exists $s \in S$ such that $s K \subseteq S$.

Proof. Since $K \cup K^{2}$ is compact, there exists $t \in T$ such that $t K \cup t K^{2} \subseteq S$. Pick any $k \in K$ and let $s=t k$. Then $s \in S$ and $s K \subseteq S$.

Lemma 2. If $p \in P$, then $P-p P$ is not a replete set.

Proof. If it were, since $\{p\}$ is compact, there would exist $p^{\prime} \in P$ such that $p p^{\prime} \in P-p P$ which is a contradiction.

Lemma 3. If $P-Q$ is replete, then $P-t Q$ is replete for all $t \in T$.

Proof. Let $t \in T$ and let $K$ be a compact subset of $T$. There exists $s \in T$ such that $s\left(K \cup t^{-1} K\right) \subseteq P-Q$. Hence $s K \subseteq P-t Q$.

Lemma 4. A set $S$ is $P$-syndetic if and only if $P \cap S$ is $P$-syndetic.

Proof. [5, Lemma 1.6]. 
Lemma 5. The following statements are equivalent.

(1) $P \cap Q$ is $P$-extensive.

(2) $P \cap Q$ is $Q$-extensive.

(3) $P \cap Q$ is replete.

(4) $P \cap Q$ is a replete semigroup.

Proof. Since (1) and (2) are symmetric and $P \cap Q$ is always a semigroup, it suffices to prove (1) equivalent to (3). We assume (1) and prove (3). Let $K$ be a compact subset of $T$. There exist $p \in P$ and $q \in Q$ such that $p K \subseteq P$ and $q K \subseteq Q$. Then $t K \subseteq P \cap Q$ for each $t \in p P$ $\cap q Q$. If $p P \cap q Q=\varnothing$, choose $p^{\prime} \in P$ so that $q p^{\prime} \in p P$ and thus $p^{\prime} P$ $\subseteq q^{-1} p P$. Hence $Q \cap p^{\prime} P=\varnothing$ and so $P \cap Q$ is not $P$-extensive, contrary to (1). Now assume (3). If (1) is false, then there exists $p \in P$ such that $(P \cap Q) \cap p P=\varnothing$. So $P-Q \subseteq P-p P$ which is not replete by Lemma 2 . Then $P-Q$ is not replete, contrary to (3).

THEOREM 1. The following statements are equivalent.

(1) $P$ and $Q$ are strongly equivalent.

(2) Neither $P-Q$ nor $Q-P$ is replete.

(3) $P$ and $Q$ have the same extensive sets.

(4) $P$ and $Q$ have the same syndetic sets.

(5) For any transformation group $(X, T, \pi)$ and any $x \in X, P_{x}=Q_{x}$.

(6) For any transformation group $(X, T, \pi)$, where $X$ is Hausdorf, and any $x \in X, P_{x}=Q_{x}$.

Proof. We establish $(1) \Rightarrow(3) \Rightarrow(2) \Rightarrow(4) \Rightarrow(1)$ and $\quad(1) \Rightarrow(5)$ $\Rightarrow(6) \Rightarrow(1)$.

First cycle. Assume (1). Let $P \cap S$ be $P$-extensive and let $q \in Q$. By the remarks at the beginning of this section there exists $p \in P$ such that $p P \subseteq q Q$. Since $S \cap p P \neq \varnothing, S \cap q Q \neq \varnothing$ and so $S \cap Q$ is $Q$-extensive. Hence $P$ and $Q$ have the same extensive sets.

Assume (3). Since $P-Q$ is clearly not $Q$-extensive, it is not $P$ extensive, so $(P-Q) \cap p P=\varnothing$ for some $p \in P$. Then $P-Q \subseteq P-p P$ which is not replete by Lemma 2 .

Assume (2). Suppose $P \cap S$ is $P$-syndetic. Since $Q-P$ is not replete, there is a compact subset $K$ of $T$ such that $P \subseteq S K$ and $t K \nsubseteq Q-P$ for any $t \in T$. Pick $q_{0} \in Q$ such that $q_{0} K \subseteq Q$. Then for each $q \in q_{0} Q$, $q K \subseteq Q$ and so $q K \cap P \neq \varnothing$. Hence $q_{0} Q \subseteq P K^{-1} \subseteq S\left(K K^{-1}\right)$. Thus $Q \subseteq S\left(q_{0}^{-1} K K^{-1}\right)$ so, by Lemma $4, S \cap Q$ is $Q$-syndetic.

Assume (4). $P$ is $P$-syndetic, hence $Q$-syndetic, so $P \cap Q$ is $Q$-syndetic and $P$-syndetic. There exists compact $K$ such that $P \subseteq(P \cap Q) K$. Since $T$ is abelian, $P \cap Q$ is $P$-extensive by an argument similar to $[3,6.17]$. Then by Lemma $5, P \cap Q$ is a replete semigroup. Choose $p \in P \cap Q$ such that $p K \subseteq P \cap Q$. Then $p P \subseteq p(P \cap Q) K \subseteq(P \cap Q)^{2} \subseteq Q$. Similarly $p Q \subseteq P$ and so $P \approx Q$. This completes the first cycle. 
To start the second cycle, notice that (5) follows easily from (1) and the definition of limit set and that (6) follows trivially from (5). We assume that (1) is false, i.e., that $P * Q$. We may assume that $P-Q$ is replete and by Lemma 1 , that $P$ is open, $Q$ is closed and $e \in Q$. Let $X=T \cup\left\{x_{0}\right\}$ and give $X$ the topology induced by the neighborhood bases inherited from $T$ along with an open neighborhood base at $x_{0}$ consisting of all sets of the form $\left\{x_{0}\right\} \cup\left(p P-q^{-1} Q\right)$ where $p \in P$ and $q \in Q$. It is easy to see that this makes $X$ a Hausdorff space. Let the phase map $\pi$ be defined to be group operation on $T \times T$ and $\pi\left(x_{0}, t\right)=x_{0}$ for all $t \in T$. We verify the continuity of $\pi$ at points $\left(x_{0}, t\right)$. Choose $t \in T$ and let $\left\{x_{0}\right\} \cup\left(p_{0} P-q_{0}^{-1} Q\right)$ be a basic open neighborhood of $x_{0}$. Let $K$ be a compact neighborhood of $e$. Then $L$ $=t K \cup t^{-1} K^{-1}$ is a compact symmetric neighborhood of $t$. It suffices to find $p \in P$ and $q \in Q$ such that $\left(p P-q^{-1} Q\right) L \subseteq p_{0} P-q_{0}^{-1} Q$. Choose $p \in P$ and $q \in Q$ so that $p L \subseteq p_{0} P$ and $q L \subseteq q_{0} Q$. Then $\left(T-q^{-1} Q\right)$ $\cap q_{0}^{-1} Q L=\varnothing$ and since $L$ is symmetric, $\left(p P-q^{-1} Q\right) L \subseteq p_{0} P-q_{0}^{-1} Q$.

In the transformation group $(X, T, \pi)$ defined by the above, $x_{0}$ is in $P_{e}$ but not in $Q_{e}$. To see the latter, notice that $\left\{x_{0}\right\} \cup P-Q$ is a neighborhood of $x_{0}$ not meeting $Q$, so $x_{0} \notin \mathrm{Cl}_{X} Q=\mathrm{Cl}_{X} \pi(e, Q) \supseteq Q_{e}$. To see that $x_{0} \in P_{e}$, let $\left\{x_{0}\right\} \cup\left(p P-q^{-1} Q\right)$ be a basic open neighborhood of $x_{0}$ and let $p_{0} \in P$. If $\left(p P-q^{-1} Q\right) \cap p_{0} P=\varnothing$, then $p p_{0} P \subseteq p P \cap p_{0} P$ $\subseteq q^{-1} Q$, so $P-q^{-1} Q \subseteq P-p p_{0} P$ which is not replete. But Lemma 3 says that $P-q^{-1} Q$ is replete. Thus $\left(p P-q^{-1} Q\right) \cap p_{0} P \neq \varnothing$ for each $p_{0} \in P$ and so $x_{0} \in \bigcap_{p \in P} \mathrm{Cl}_{X} p P=\bigcap_{p \in P} \mathrm{Cl}_{X} \pi(e, p P)=P_{e}$. Hence (6) is false and the proof is complete.

Theorem 1 guarantees that strongly equivalent replete semigroups will have the same limit sets for any transformation group $(X, T, \pi)$. However, it is easy to think of transformation groups where $P_{x}=Q_{x}$ for each $x \in X$ although $P$ is not strongly equivalent to $Q$. The next theorem shows that for each locally compact abelian group $T$, there is an action of $T$ on a space $X$ and a point $x \in X$ such that inequivalent replete semigroups have unequal limit sets. More precisely, we have

Theorem 2. For every locally compact abelian topological group $T$, there is a transformation group $(X, T, \pi)$ and a distinguished point $x \in X$ such that $P \approx Q$ if and only if $P_{x}=Q_{x}$.

Proof. Let $[P]$ denote the equivalence class containing $P$. Let

$$
X=T \cup\{([P],[Q]) \mid P \text { open, } Q \text { closed, } e \in Q, P-Q \text { replete }\}
$$

and give $X$ the following topology: as in Theorem 1, the points of $T$ 
inherit their neighborhood systems from $T$. Points $([P],[Q])$ have an open neighborhood base consisting of all sets of the form $\{([P],[Q])\} \cup\left(p P-q^{-1} Q\right)$ where $p \in P$ and $q \in Q$. The only thing which needs verifying is that this is well defined. So let $P \approx P_{1}$ and $Q \approx Q_{1}$ and let $\{([P],[Q])\} \cup\left(p P-q^{-1} Q\right)$ be a basic open neighborhood of $([P],[Q])$. Choose $p_{1} \in P_{1}$ so that $p_{1} P_{1} \subseteq p P$. There exists $q^{\prime} \in Q_{1}$ such that $q^{\prime} Q_{1} \subseteq q Q_{1} \cap Q_{1}$. Choose $q^{\prime \prime} \in Q$ so that $q^{\prime \prime} Q \subseteq q^{\prime} Q_{1}$. Then $q_{1}=q^{\prime \prime} q^{\prime \prime} \in Q_{1}$ and $q_{1} Q \subseteq q Q_{1}$. Hence $p_{1} P_{1}-q_{1}^{-1} Q_{1} \subseteq p P-q^{-1} Q$.

Define the phase map $\pi$ to be group operation on $T \times T$ and to leave all the ideal points fixed. Then, just as in Theorem 1, $([P],[Q])$ is in $P_{e}$ hut not $Q_{e}$.

REMARK. It is easy to see that there are only two strong equivalence classes of replete semigroups in $R^{1}$. In $R^{n}$ where $n \geqq 2$, things change drastically. Hahn showed in [4] that every replete semigroup in $R^{n}$ contains a wedge and that every wedge is itself a replete semigroup. It is immediate that any wedge in $R^{n}$ is strongly equivalent to a closed wedge with vertex at the origin and that any two closed wedges with vertex at the origin are strongly equivalent if and only if they are identical. Thus for $n \geqq 2$, the number of strong equivalence classes in $R^{n}$ is uncountable.

3. Weak equivalence. In this paragraph we study an equivalence relation among replete semigroups which will guarantee that $P(x)$ $=Q(x)$ precisely when $P$ and $Q$ are equivalent. The situation is parallel to that studied in §2, so many of the details will be omitted.

We say that $P$ and $Q$ are weakly equivalent and write $P \sim Q$ provided that given replete semigroups $P^{\prime} \subseteq P$ and $Q^{\prime} \subseteq Q$, there exist replete semigroups $P^{\prime \prime} \subseteq P$ and $Q^{\prime \prime} \subseteq Q$ such that $P^{\prime \prime} \subseteq Q^{\prime}$ and $Q^{\prime \prime} \subseteq P^{\prime}$.

It is easy to see that this is an equivalence relation.

THEOREM 3. The following statements are equivalent.

(1) $P$ and $Q$ are weakly equivalent.

(2) Neither $P-Q$ nor $Q-P$ contains a replete semigroup.

(3) $P$ and $Q$ have the same extensive replete semigroups.

(4) $P$ and $Q$ have the same weakly extensive sets.

(5) For any transformation group $(X, T, \pi)$ and any $x \in X, P(x)$ $=Q(x)$.

(6) For any transformation group $(X, T, \pi)$ where $X$ is Hausdorff and any $x \in X, P(x)=Q(x)$.

Proof. It is easy to check that $(1) \Rightarrow(3) \Rightarrow(2) \Rightarrow(4) \Rightarrow(1)$. It is also easy to see that $(1) \Rightarrow(5) \Rightarrow(6)$. So assume (1) is false. Note that the first cycle shows that strong equivalence implies weak equivalence. 
So we may assume that $P \nsim Q$ and that $Q$ is open and $P-Q$ contains a replete semigroup. Adjoin to $T$ the ideal point $x_{0}$ with a base of open neighborhoods consisting of all sets of the form $\left\{x_{0}\right\} \cup_{q Q}$ where $q \in Q$. Let $T$ fix the ideal point and translate by group operation the other points. It is easy to see that $P(e)=\varnothing$ and that $Q(e)=\left\{x_{0}\right\}$, so that (6) is false.

Theorem 4. For every locally compact abelian topological group $T$, there is a transformation group $(X, T, \pi)$ and a distinguished point $x \in X$ such that the following statements are equivalent.

(1) $P$ and $Q$ are weakly equivalent.

(2) $P_{x}=Q_{x}$.

(3) $P(x)=Q(x)$.

Proof. Let $X=T \cup\{[P]\}$ and give $X$ the following topology: the points of $T$ inherit their neighborhood bases from $T$ and an ideal point $[P]$ has an open neighborhood base consisting of all sets of the form $\{[P]\} \cup Q$ where $Q$ is open and $P \approx Q$. That this defines a topology follows from the easily verified fact that if $Q_{1}$ and $Q_{2}$ are in $[P]$, then so is $Q_{1} \cap Q_{2}$. Again define a transformation group $(X, T, \pi)$ by translating points of $T$ and leaving all ideal points fixed. The distinguished point is $e$.

4. Comparison of equivalence relations. We have noted that it follows from Theorems 1 and 3 that strong equivalence implies weak equivalence. The converse is false as the following example shows. In the plane, let $P=\{(s, t) \mid s, t \geqq 1\}$ and let $Q=\{(s, t) \mid s \geqq 1$ and $t \geqq \sqrt{ } s\}$. Then $P-Q$ is replete because $\sqrt{ } s \rightarrow+\infty$ as $s \rightarrow+\infty$, but $P-Q$ contains no wedge, hence no replete semigroup. Thus $P \sim Q$ but $P \approx Q$. Notice that $Q$ is $Q$-syndetic but not $P$-syndetic. This explains the absence from Theorem 3 of a condition about common syndetic replete semigroups for $P$ and $Q$ weakly equivalent.

\section{REFERENCES}

1. John D. Baum, P-recurrence in topological dynamics, Proc. Amer. Math. Soc. 7 (1956), 1146-1154.

2. Ethan M. Coven, P-recursion and transformation groups having an equicontinuous replete semigroup, Math. Systems Theory (to appear).

3. W. H. Gottschalk and G. A. Hedlund, Topological dynamics, Amer. Math. Soc. Colloq., Publ., vol 36, Amer. Math. Soc., Providence, R. I., 1955.

4. Frank J. Hahn, Some embeddings, recurrence properties, and the BirkhoffMarkov theorem, Duke Math. J. 27 (1960), 513-525.

5. William L. Reddy, Almost periodic semigroups in transformation groups, Illinois J. Math. 12 (1968), 494-509.

WESLEYAN UNIVERSITY 